\title{
Guanxinjing capsule in the treatment of chronic stable angina: study protocol for a randomized controlled trial
}

Ying Tian ${ }^{1,2}$, Junhua Zhang ${ }^{1}$, Yingqiang Zhao ${ }^{2}$, Jingyuan Mao ${ }^{3}$, Linping Zhu², Rui Gao ${ }^{4}$, Xuemei Wang ${ }^{5}$, Mingjun Zhu ${ }^{6}$, Lihong $\mathrm{Ma}^{7}$, Mengyu Zhao ${ }^{1}$ and Wenke Zheng ${ }^{1 *}$

\begin{abstract}
Background: Stable angina is a common cardiovascular disease with high mortality and a poor prognosis. Although there are various interventions against stable angina, none are able to significantly reduce the mortality rate. Guanxinjing capsule (GXJ) is made from the classical Chinese prescription Xuefuzhuyutang (血府逐病汤). Both basic research and clinical studies have shown that GXJ can relieve the symptoms of angina, but currently, the effects of GXJ lack high-quality clinical evidence. The aim of this study was to evaluate the clinical effectiveness and safety of GXJ compared with placebo.

Methods/design: This multicentre, blinded, randomized trial will be conducted with a total of 120 participants diagnosed with chronic stable angina (Qi deficiency and blood stasis syndrome). Using a central randomization system, participants will be randomized (1:1) into groups receiving either GXJ or placebo for 8 weeks. After a 2week run-in period, eligible patients will receive either GXJ or placebo (4 pills, three times daily) for 8 weeks in addition to conventional treatment. The primary outcomes include changes in the total exercise time on exercise tolerance tests and changes in the integral scores of angina symptoms. The secondary outcome measures include changes in the maximal estimated workload, changes in time to a $1 \mathrm{~mm}$ ST-segment depression or raise, changes in the time to onset of angina during exercise tolerance testing, changes in the total score of traditional Chinese medicine syndrome, and changes in the total score of the Generalized Anxiety Disorder 7-item assessment between baseline and week 8. Other outcome measures will also be assessed. All exercise tolerance tests use a standard Bruce multistage exercise test protocol. Adverse events will be monitored throughout the trial.
\end{abstract}

Discussion: This study will investigate whether GXJ can alleviate clinical symptoms, increase the angina-free walking time, and improve quality of life in patients with chronic stable angina (Qi deficiency and blood stasis syndrome). The results of this study will provide clinical evidence for the application of GXJ to the treatment of stable angina.

Trial registration: Chinese Clinical Trial Registry, ChiCTR1800014258. Registered on 2 January 2018.

Keywords: Chinese herbal medicine formula, Angina pectoris, Qi deficiency and blood stasis syndrome, Guanxinjing capsule, Blinded, randomized controlled trial

\footnotetext{
* Correspondence: zhengwk@tice.com.cn

${ }^{1}$ Tianjin University of Traditional Chinese Medicine, 312 Anshanxi Road,

Nankai District, Tianjin 300193, China

Full list of author information is available at the end of the article
}

(c) The Author(s). 2018 Open Access This article is distributed under the terms of the Creative Commons Attribution 4.0 International License (http://creativecommons.org/licenses/by/4.0/), which permits unrestricted use, distribution, and reproduction in any medium, provided you give appropriate credit to the original author(s) and the source, provide a link to the Creative Commons license, and indicate if changes were made. The Creative Commons Public Domain Dedication waiver (http://creativecommons.org/publicdomain/zero/1.0/) applies to the data made available in this article, unless otherwise stated. 


\section{Background}

Stable angina is a clinical syndrome characterized by discomfort in the chest, jaw, shoulder, back, or arms, typically elicited by exertion or emotional stress and relieved by rest or nitroglycerin [1]. Stable angina is a common and disabling disorder, and the prevalence is $3.6 \%$ in China [2]. Data from the literature report that the incidence of angina in the USA is 12.3 cases/1000 person years (age-adjusted, ages 45-74 years), with a prevalence of $20 \%$ in men older than 60 years, and similar numbers have been reported in Europe [3, 4]. The annual death rate of patients with stable angina is $1.2-2.4 \%$ [5]. This syndrome poses a large burden on the healthcare system and calls for continued research and the development of therapies, as well as prevention.

Chinese medicines have been used in therapeutic approaches in East Asia for more than two millennia. In Chinese medicine, angina pectoris is classified as "XiongBi" or "heartache" according to their symptoms, with a record dating from the Han dynasty and the accumulation of many effective treatments. In China, Chinese patent medicines are prevalent and are commonly used as an alternative to Western medicine to treat coronary heart disease (CHD) [6, 7]. Chinese medicines will be the optimal choice of complementary and alternative medicines for the treatment of angina pectoris.

From the perspective of traditional Chinese medicine (TCM), patients with CHD can be divided into different syndromes (Zheng), which is a summarization of the pathological changes that characterize the disease based on the location, the cause and nature of the disease, and the states of Xie (pathogenic factors) and Zheng (the healthy Qi). According to the data from a previous research regarding the syndrome differentiation of chronic stable angina, the syndrome of "Qi deficiency and blood stasis" might reach a proportion of $18.62 \%$ of clinical patients, which would rank first among the different subtypes [8]. A meta-analysis showed that reinforcing the Qi and activating the blood effectively ameliorates angina symptoms and demonstrates a safety profile among patients with $\mathrm{CHD}[9,10]$.

In TCM theory, Qi represents a function. If the Qi is abundant, the function will be powerful, while if the Qi is insufficient, the function will be weak.

The Guanxinjing capsule (GXJ) was approved by the China Food and Drug Administration for the treatment of CHD in 2002. GXJ is based on a famous traditional Chinese prescription, Xuefuzhuyutang (血府逐瘀汤), which was created by Wang Qinren, a famous physician of the Qing dynasty, and has been widely used to treat various diseases caused by stasis. The GXJ capsule is composed of salvia, rhizomaligusticichuanxiong, red peony root, safflower, ginseng, polygonatum, Panax notoginseng, storax, and borneol, and functions to reinforce the Qi and activate the blood. Several studies have suggested that GXJ can increase myocardial hypoxia tolerance [11] and relieve the clinical symptoms of angina pectoris [12]. However, these studies were performed decades ago, with low-quality methods. In this study, we designed a prospective, randomized, multicentre, double-blind, placebo-controlled trial to investigate the effectiveness and safety of GXJ in adults with chronic stable angina, with the aim of providing powerful evidence for clinical application. This study is registered in the Chinese Clinical Trial Registry (ChiCTR1800014258).

In addition, depression and anxiety in patients with cardiac disease is highly prevalent and is independently associated with adverse cardiovascular consequences, including worse health-related quality of life, impaired functional status, recurrent cardiac events, and higher rates of mortality [13-17]. People with stable angina, an important subtype of cardiac disease, experience difficulties similar to those experienced by patients with other types of CHD, including depression, anxiety, and impaired quality of life [18-20]. Although studies found that antidepressant drug treatment did not improve the cardiovascular prognosis [21-23], we must consider the early psychological problems of patients with stable angina. Previous studies have suggested that GXJ can increase the concentration of 5-hydroxytryptamine (5-HT) and improve behavioural performance in depressed rats after heart attacks [24]. GXJ was recommended in the Consensus on the Diagnosis and Treatment of Depression before and after Percutaneous Coronary Intervention (PCI) [25] for the treatment of depression and/or anxiety before and after PCI. The Generalized Anxiety Disorder 7-item (GAD-7) test (see Additional file 1) is one of the few GAD measures that is also specifically linked to the Diagnostic and Statistical Manual of Mental Disorders, $4^{\text {th }}$ edition (DSM-IV) criteria. The GAD-7 test is a reliable tool for anxiety screening and diagnosis, as it uses patient self-evaluation, is easy to use, and has good reliability and validity in cardiovascular patients [26]. A score of 10 or higher on the GAD-7 test represents a reasonable cut point for identifying cases of GAD. Cut points of 5,10 , and 15 might be interpreted as representing mild, moderate, and severe levels of anxiety on the GAD-7 test. Construct validity was demonstrated by the fact that increasing scores on the GAD-7 scale were strongly associated with multiple domains of functional impairment [27]. In this study, we choose to use the GAD-7 scale as a screening tool to evaluate patient mental health to observe the effects of GXJ on patient mental states.

Based on the multifactorial effects of GXJ on CHD, our hypothesis is that GXJ, in addition to conventional treatments, can provide relief of angina symptoms in patients with chronic stable angina pectoris (Qi deficiency and blood stasis syndrome) when compared with 
conventional treatment alone. If successful, GXJ will represent a novel, promising alternative strategy for the further relief of angina symptoms.

\section{Objective}

This study will evaluate the effectiveness and safety of the GXJ in adults with chronic stable angina pectoris (Qi deficiency and blood stasis syndrome) to clarify the role of GXJ in the medical field.

\section{Methods/design}

\section{Study design}

This study is a prospective, randomized, multicentre, blind, placebo-controlled trial.

The study is registered in the Chinese Clinical Trial Registry (ChiCTR1800014258) and complies with the principles of the Declaration of Helsinki and Good Clinical Practice (GCP) guidelines. Written informed consent will be obtained from all patients prior to their participation in this study, and the recruited patients will be randomly assigned to either the GXJ group or the placebo group. The protocol design is based on the following Standard Protocol Items: Recommendations for Interventional Trials (SPIRIT) checklist (see Additional file 2). We will rigorously follow the Consolidated Standards of Reporting Trials (CONSORT) Extension for Chinese Herbal Medicine Formulas 2017 recommendations in reporting the results [28].

The trial will be conducted at eight centres in China (see Additional file 3), and a total of 120 participants will be recruited. After the participants have provided consent, they will be enrolled in the trial, which consists of a 2-week run-in period and an 8-week treatment period. A flow diagram of the study procedures is illustrated in Fig. 1.

\section{Recruitment}

Participants will be recruited through Internet advertisement and posters in the community and selected hospitals.

\section{Eligibility criteria}

Eligible patients are those who meet all of the following inclusion criteria (listed in a subsequent section) and who do not have any of the listed exclusion criteria.

\section{Diagnostic criteria}

The diagnostic criteria and definitions are listed as follows.

1. The diagnostic criteria for CHD are (1) a history of myocardial infarction, with or without revascularization (PCI or coronary artery bypass grafting) treatment; (2) coronary angiography or computed tomography coronary angiography confirmation of stenosis greater than $50 \%$ of at least one major branch of the coronary artery luminal diameter, with or without revascularization; or (3) noninvasive imaging stress test diagnostic of coronary artery disease (CAD) (e.g. nuclear perfusion scan, stress echocardiogram, or stress cardiac magnetic resonance scan). A patient meeting at least one of these criteria is considered to be diagnosed with CHD.

2. The diagnostic criteria for chronic stable angina were determined according to the guidelines for the diagnosis and treatment of chronic stable angina of China in 2007 [29], the 2013 ESC guidelines on the management of stable coronary artery disease [30], and the SIGN guideline on coronary revascularisation in the management of stable angina pectoris [31]. The classification of angina referred to the Canadian Cardiovascular Society (CSS) Functional Classification of Angina [32] (see Additional file 4).

3. The definition of major adverse cardiovascular event (MACE) is as follows: cardiac death (any death, unless an unequivocal noncardiac cause can be established), nonfatal myocardial infarction (MI) (appearance of pathological Q waves that were absent at baseline or a total creatine kinase [CK] level more than two times the upper limit of normal [ULN], with the presence of CK isoenzyme MB at greater levels than the ULN), or urgent revascularization with either PCI or coronary artery bypass graft.

\section{Standardization of traditional Chinese medicine differentiation}

According to the technical guidelines for clinical research of TCM drugs and natural drugs treated for angina and coronary artery disease (2011) [33], the primary signs and symptoms are chest congestion and chest pain. Secondary signs and symptoms are palpitation, shortness of breath, fatigue, loss of energy, and a dark and purple complexion. In addition, the patient has a slight purple tongue and a weak and uneven pulse.

A patient with one of the primary symptoms and two of the secondary symptoms, combined with the tongue and pulse conditions, can be diagnosed as having Qi deficiency and blood stasis syndrome.

\section{Inclusion criteria}

The inclusion criteria for the trial are as follows:

1. A diagnosis of $\mathrm{CHD}$ and the provision of exact imaging information (coronary angiography or computed tomography coronary angiography 


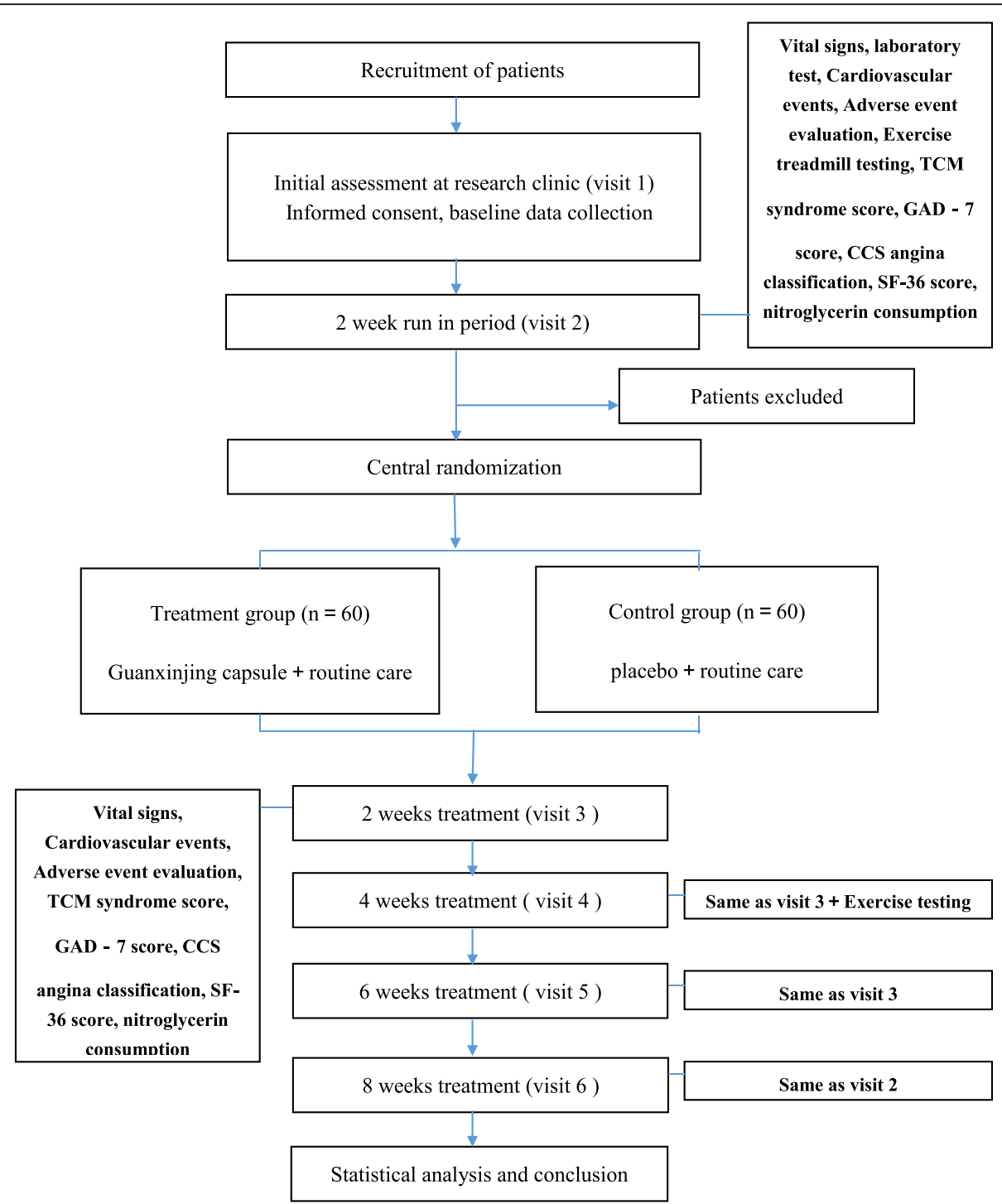

Fig. 1 Flow diagram

confirmation of stenosis greater than $50 \%$ of at least one major branch of the coronary artery luminal diameter, or nuclear perfusion scan diagnosed as CAD). CCS classification of angina grade II

2. TCM diagnosis of Qi deficiency and blood stasis syndrome, according to TCM standards

3. At least 2 months of standardized medication history before enrolment (sustaining the same medication, type, usage, and dose)

4. Age between 18 and 75, either male or female

5. Signed informed consent by participants or surrogates

6. Understanding and voluntary compliance with the research program, acceptance of the prescribed course and limits of the study, understanding the oral scale and diary card

\section{Exclusion criteria}

The exclusion criteria for the trial are as follows:

1. Uncontrolled hypertension (systolic blood pressure $\geq 160 \mathrm{mmHg}$ or diastolic blood pressure $\geq$ $100 \mathrm{mmHg}$ ). Severe cardiopulmonary insufficiency, or severe arrhythmia (rapid atrial fibrillation and flutter, paroxysmal ventricular tachycardia second degree and greater than a second degree atrioventricular $[\mathrm{AV}]$ block). Acute $\mathrm{MI}$ in the past 2 months, or has undergone coronary revascularization in the past 12 months

2. Renal insufficiency, male serum creatinine $>2.5 \mathrm{mg} /$ $\mathrm{dl}(>220 \mu \mathrm{mol} / \mathrm{L})$ or female serum creatinine $>$ $2.0 \mathrm{mg} / \mathrm{dl}(>175 \mu \mathrm{mol} / \mathrm{L})$. Serious liver disease (alanine transaminase and/or aspartate 
transaminase values two times higher than the upper reference limit value). Other severe primary disease, such as malignant tumour and haematopoietic system disease

3. Complications with absolute contraindications or relative contraindications during exercise tolerance testing (ETT) (e.g. severe aortic stenosis, acute aortic dissection, left primary coronary artery stenosis, acute myocarditis, or pericarditis)

4. Factors that precluded satisfactory interpretation of the electrocardiogram (ECG) (e.g. digoxin therapy, left bundle branch block, implanted with pacemaker, left ventricular hypertrophy, or electrolyte disturbance)

5. Complications with a serious bone joint disease or other comorbidities that may interfere with ability to perform required ETT

6. Patients planning to undergo coronary revascularization during the study period

7. Patients who might be allergic or are known to be allergic to ingredients of the study drug

8. Women who are pregnant or trying to become pregnant or are in the middle of a lactation period

9. Substance abuse, alcohol and drug dependence in the last 2 years

10. Other conditions that may affect the subjects' adherence or lead to safety problems in the study

11. Participation in other clinical trials within the past 30 days

\section{Other criteria}

\section{Permitted and prohibited drugs}

The following criteria apply to permitted and prohibited drugs:

1. Chinese herbs, drugs, and non-drug therapy which might alleviate angina or improve myocardial energy metabolism or upset restless symptoms are prohibited to use, including benzodiazepine sedatives and serotonin reuptake inhibitors.

2. Patients will be instructed to take a nitroglycerin tablet ( $0.5 \mathrm{mg}$ per tablet) in case of angina pectoris, and this dose can be repeated approximately every 5 min until the angina is relieved. If the angina persists after three doses, the patient should be transported to a hospital immediately for further medical treatment. Patients will be asked to record the details of administration times, the number of doses, and the dosages of nitroglycerin in a patient diary, which will be collected by the investigators at a subsequent study visit.

Concomitant medications that are deemed necessary to manage any serious underlying diseases are allowed during the study. The names and amounts of concomitant medications should be recorded in detail in the patients' medical records and case report forms (CRFs).

\section{Suspension criteria}

The criteria for suspension of participation are as follows:

1. Occurrence of serious adverse events (AEs), complications, or fatal physiological changes

2. Contravention of research program, such as the use of forbidden drugs prescribed by the program or not taking the medicine according to the research program (poor compliance)

3. Voluntary withdrawal

4. Withdrawal for various reasons, such as death or failure to attend follow-up visits

5. Incomplete data

\section{Interventions}

\section{Methods of administration}

Eligible patients will be allocated to a treatment group or a control group. During the 2-week run-in period, all subjects will receive placebo capsules in addition to routine medications. Then, during the treatment period, eligible patients will be given GXJ capsules (4 pills, three times daily; China State Food and Drug Administration [SFDA] approval number Z20025812; composed of salvia, rhizomaligusticichuanxiong, red peony root, safflower, ginseng, polygonatum, Panax notoginseng, storax, and borneol) or placebo for 8 weeks, in addition to conventional treatment, which will maintain the same dosage. The GXJ and placebo capsules are provided by Baoding Traditional Chinese Medicine Pharmaceutical Co. Ltd, Anguo, China. Analyses have shown that the quality of the GXJ capsules is consistent with the Chinese Medicine Standards of the SFDA. The placebo capsule is similar to the GXJ capsule, with a comparable appearance. Each capsule is $0.3 \mathrm{~g}$. The primary content of the placebo capsule is starch. By adding food colourants and flavouring agents, the mixture achieves a colour, smell, taste, and texture comparable to the contents of the GXJ capsule.

\section{Routine medications}

Routine medications in this trial include antiplatelet medications, lipid-lowering medications, angiotensinconverting enzyme inhibitors or angiotensin receptor blockers, $\beta$-blockers, and calcium channel blockers, which must be maintained at the same dosage as prior to recruitment.

\section{Sample size}

This is a clinical pilot trial; therefore, the total sample size of this study has been preliminarily determined to 
be 120 (during the trial, the expulsion rate is controlled within $20 \%$ ) on expert advice. The treatment group and the control group will contain the same number of cases (that is, there will be 60 cases in each group).

\section{Randomization, allocation concealment mechanism, and blinding}

Participants will be randomized in a 1:1 ratio, using a computer-generated, site-stratified, block randomization schedule. Randomization of the trial participants will be completed using an independent data centre (Tianjin Institute of Clinical Evaluation). A number will be placed in an envelope and subsequently sealed. The statistician will send the envelope directly to the product manufacturer for the labelling of the active and placebo products, according to the randomization number. The study capsules, labelled with sequential randomization numbers, will be sent to each research centre, and each patient will be assigned the lowest number available at each participating centre. All patients, care providers, and attending physicians will be blinded to the treatment assignments until the study is completed. Duplicated blinding codes will be provided to the primary research institution and the manufacturer to maintain, and the blinding codes cannot be broken until all the clinical data are entered into an EpiData database and locked, except in an emergency situation.

\section{Emergency unblinding}

Only if a subject experiences an $\mathrm{AE}$ and the drug must be identified immediately can the unblinding letter be disassembled by the major investigator of the research unit. The reason for the unblinding will then be reported to the inspector. The subjects are withdrawn from the study after unblinding. Details of the unblinding cause, date, treatment situation, and results will be reported in the CRF and signed by the administrator.

\section{Content and points of data capture}

The content and points of data capture in the trial are as follows:

1. Run-in period (14 days): 14 days before recruitment

2. Intervention period ( 8 weeks): follow-up every 2 weeks and recorded

Different items are measured according to the time points of data collection. The details are shown in Table 1.

\section{Outcome measurements Primary outcomes}

The primary outcomes of the study include change in total exercise time (time frame: baseline [visit 2] and week 8 [visit 6]) of ETT and the score of the angina symptom integral. Total exercise time was defined as the maximal duration of exercise that was performed by a patient in the ETT setting. All ETT tests used a standard Bruce multistage exercise test protocol. The angina symptom integral includes four contents: the severity of angina, the frequency of angina, the duration of angina, and the nitroglycerin dosage. The total score can range from 0 to 24, and higher scores indicate a worse status (see Table 1).

\section{Secondary outcomes}

The secondary outcome measures include:

1. Changes in maximal estimated workload (in metabolic equivalents [METS]) during ETT between baseline and week 8

2. Changes in time to $1 \mathrm{~mm} \mathrm{ST-segment} \mathrm{depression}$ or raise during ETT at the end of the treatment period

3. Changes in time to onset of angina during ETT at the end of the treatment period

4. The total score of the TCM syndrome

5. The total score of the GAD-7 test

\section{Other outcome measures}

Other outcome measures include the following:

1. A 36-item Short Form Health Survey (SF-36) score

2. Consumption of short-acting nitrates

3. Angina grade, according to the CCS Angina Grading Scale

4. The probability of a MACE occurrence

\section{Safety outcomes}

Safety outcomes includes vital signs (temperature, heart rate, breathing, and blood pressure after $10 \mathrm{~min}$ of rest), a complete blood count, urine and stool tests, kidney and liver function tests, coagulation function test, and an evaluation of the occurrence of AEs.

\section{Adverse events}

Every AE occurring during the study must be recorded in the CRF, according to the actual circumstances. The following information should be recorded: occurrence time, severity, duration, adopted measure, and outcome of the AE. The researcher should evaluate the correlation between the $\mathrm{AE}$ and the drug and decide whether to stop the observation, according to the condition, and follow-up investigations should be conducted on the cases that are discontinued due to adverse reactions. 
Table 1 Measurement items and points of data capture

\begin{tabular}{|c|c|c|c|c|c|c|}
\hline \multirow[t]{2}{*}{ Study phase } & \multirow{2}{*}{$\begin{array}{l}\text { Run-in period } \\
\text { Visit } 1\end{array}$} & \multicolumn{5}{|c|}{ Intervention period } \\
\hline & & Visit 2 & Visit 3 & Visit 4 & Visit 5 & Visit 6 \\
\hline Time & -14 days & 0 day & 2 weeks & 4 weeks & 6 weeks & 8 weeks \\
\hline \multicolumn{7}{|l|}{ Baseline data collection } \\
\hline Informed consent & $\times$ & & & & & \\
\hline Inclusion/exclusion criteria & $x$ & & & & & \\
\hline Demographic data & $\times$ & & & & & \\
\hline Get the central random number & $\times$ & & & & & \\
\hline Concomitant disease and treatment & $\times$ & & & & & \\
\hline Previous history, medical history and allergies & $x$ & & & & & \\
\hline \multicolumn{7}{|l|}{ Safety evaluation } \\
\hline Vital signs & $\times$ & $\times$ & $\times$ & $\times$ & $\times$ & $\times$ \\
\hline Complete blood count, urine and stool tests & & $x$ & & & & $\times$ \\
\hline Liver and renal function tests & & $\times$ & & & & $\times$ \\
\hline Coagulation function test & & $x$ & & & & $\times$ \\
\hline Cardiovascular events & $\times$ & $\times$ & $\times$ & $x$ & $\times$ & $\times$ \\
\hline Adverse event evaluation & $\times$ & $x$ & $\times$ & $x$ & $\times$ & $\times$ \\
\hline \multicolumn{7}{|l|}{ Efficiency evaluation } \\
\hline Exercise treadmill testing' & & $x$ & & $x$ & & $x$ \\
\hline TCM syndrome score & $\times$ & $x$ & $\times$ & $x$ & $\times$ & $\times$ \\
\hline GAD-7 score & $\times$ & $x$ & $\times$ & $x$ & $\times$ & $\times$ \\
\hline CCS angina classification & $\times$ & $x$ & $\times$ & $x$ & $\times$ & $\times$ \\
\hline SF-36 score & $\times$ & $x$ & $\times$ & $x$ & $\times$ & $\times$ \\
\hline Nitroglycerin consumption & $\times$ & $x$ & $\times$ & $x$ & $\times$ & $\times$ \\
\hline \multicolumn{7}{|l|}{ Other work } \\
\hline Concomitant medications & $\times$ & $x$ & $\times$ & $x$ & $\times$ & $\times$ \\
\hline Dispense drug & $\times$ & $x$ & $\times$ & $x$ & $\times$ & \\
\hline Recovery and record of study drug & & $x$ & $\times$ & $\times$ & $\times$ & $\times$ \\
\hline Evaluate the adherence & & $\times$ & $\times$ & $\times$ & $\times$ & $\times$ \\
\hline Study completion status & & & $\times$ & $\times$ & $\times$ & $\times$ \\
\hline CRF examination & & & & & & $x$ \\
\hline
\end{tabular}

Abbreviations: SF36 36-item Short Form Health Survey, TCM traditional Chinese medicine, CCS Canadian Cardiovascular Society, GAD-7 Generalised Anxiety Disorder Assessment, CRF Case Report Form

\section{Quality control of the intervention}

Quality control will be conducted by rigorous monitoring throughout the trial. All of the staff, including the operators, investigators, data collectors and analysers, are to be strictly trained to ensure that all practices at each hospital are generalized and standardized according to the standard operating procedures. Physicians must pass the required training test to demonstrate an understanding of the purpose and content of the trial, intervention strategies, and any $\mathrm{AE}$ observations. A multicentre test coordination committee, with a general manager of clinical research, will be established and will be responsible for the coordination of the clinical trials and for solving the relevant problems. The organizer will appoint monitors who will visit the clinical centres regularly to ensure that the records of the trial are complete and accurate, as well as to supervise the implementation of clinical trial programs.

The ETT method refers to the 2013 American Heart Association exercise standards for testing and training [34]. Eligible patients enter a single-blind, placebo-treatment, qualifying phase, during which they undergo Bruce [34] exercise treadmill tests. A standard 12-lead ECG with the patient in a supine position is obtained before each exercise treadmill test, and torso ECGs with the patient standing are monitored throughout the exercise testing. A core ECG laboratory (Tianjin University of TCM), blinded to treatment assignment, will interpret all rest and 
exercise ECGs. Exercise-induced ECG ischaemia is defined as the new development of horizontal or down-sloping ST-segment depression $(1 \mathrm{~mm}$ at $80 \mathrm{~ms}$ after the J point) compared to baseline tracing. For patients with a permitted baseline ST-depression at rest $(1 \mathrm{~mm})$, qualifying ST-segment depression is defined as additional ST depression of at least $1 \mathrm{~mm}$ below the resting value. Subsequent exercise tests are performed approximately $2 \mathrm{~h}$ after morning dosing at 4 and 8 weeks after randomization.

At each visit, the investigators will record the number of pills the patients receive, use, and return, in detail, and record this information on the CRF without delay to evaluate the adherence of the subjects. Unused study drugs are counted. Drug compliance should be between 80 and $120 \%$, with compliance $=$ practical dose $/$ applica tion dose $\times 100 \%$. The diary of angina episodes listed by the patient will be retrieved and maintained after the study. To maximize compliance and retention throughout the study, all participants will receive a telephone call before each follow-up appointment to remind them of the treatment time and location. Additionally, we will attempt to prevent dropouts by providing ongoing support to participants in the form of transport allowance, free medical examinations related to the research.

\section{Statistical analysis}

Statistical analysis will be performed by the Tianjin Institute of Clinical Evaluation, and the data will be analysed using Statistical Analysis System (SAS) software version 9.3 (SAS Institute Inc., Cary, NC, USA). All effectiveness and safety analyses will be strictly conducted according to the intention-to-treat (ITT) principle, the full analysis set (FAS) population, the per protocol set (PPS) population, and the safety set (SS) population. Continuous variables will be described using means and standard deviations and tested with Student's $t$ tests. Categorical variables will be presented using percentages and tested with chi-squared tests. More details will be described in a formal statistical analysis plan.

\section{Data management}

The patient's information will be added to the paper CRF by researchers and reviewed by the clinical examiner. The first copy of the CRF will then be forwarded to the data administrator for data entry and management. To ensure the accuracy of the data, two data entry staff should independently input the data, and the data should be checked twice.

Data lockup will be implemented after the blinding state data review has confirmed the data to be correct. Researchers will be unable to modify data after locking.

\section{Ethical issues}

\section{Ethics statement}

Researchers are responsible for ensuring that the study is conducted in accordance with the principles of the Declaration of Helsinki and GCP. Participants will voluntarily provide their written informed consent before any study procedures, and they can voluntarily withdraw from the study for any reason.

\section{Ethical approval}

This trial protocol was approved by the Ethics Committee of the Tianjin University of TCM (TJUTCM-EC20170006). The study protocol and informed consent procedure are consistent with scientific and ethical requirements. Written informed consent must be obtained from all participants or their legally authorized representatives before enrolment.

\section{Protocol amendments}

If there are any problems during the implementation of the plan and it becomes necessary to revise it, the revised plan will be submitted to the ethics committee for approval before implementation.

\section{Discussion}

Atherosclerotic cardiovascular disease (CVD) is a chronic disorder that develops insidiously throughout life and usually has progressed to an advanced stage by the time symptoms occur, such as angina. According to World Health Organization statistics, the annual global CVD mortality is predicted to be 23.6 million by 2030 , and more people die annually from CVD than from any other cause, with an estimated 17.5 million deaths in 2012 (46\% of all noninfectious chronic disease [NCD] deaths) [35]. Although a number of Western medications have been effective in reducing all-cause or CVD mortality, CVD remains a problem everywhere. Many patients do not receive conventional treatments due to side effects, contraindications, and drug-drug interactions [30], and many still experience angina and ischaemia at low workloads, despite undergoing standard treatments. Therefore, a broader range of therapeutic agents than are currently available is required to improve the quality of life for these patients.

TCM has been used for thousands of years to treat diseases. Many classic formulas have been modified by modern techniques and have been applied in clinical practice. Recently, there is a growing and sustained interest in the benefits of TCM and potential drug interactions with Western medications, especially for patients with stable angina. GXJ is a type of Chinese patent medicine that is used to treat Qi deficiency and blood stasis syndromes. The primary ingredients are salvia (Danshen), rhizomaligusticichuanxiong (Chuanxiong), 
red peony root (Chishao), safflower (Honghua), ginseng (Renshen), polygonatum (Yuzhu), Panax notoginseng (Sanqi), storax (Suhexiang), and borneol (bingpia), with the functions of reinforcing the Qi and activating the blood. Qi deficiency and blood stasis provide the crucial pathogenesis of stable angina in the TCM concept. We designed this trial with the hope of verifying that GXJ plus conventional treatment can provide relief of angina symptom in patients with chronic stable angina pectoris (Qi deficiency and blood stasis syndrome). If successful, this treatment will represent a novel, promising, alternative strategy for further relieving angina symptoms.

The study design has two primary points. (1) This is a superiority trial comparing a study drug with placebo, selecting chronic stable angina patients with exact imaging information. (2) By adopting the clinical effect evaluation methods of "the combination of disease and syndrome, multidimension index" [36], we selected indices that reflect both the advantages and characteristics of TCM and the conventional efficacy evaluation of Western medicine, setting the changes in total exercise time during ETT tests and the changes in the integral score of angina symptoms as the primary outcomes and the total score of TCM syndrome as the secondary outcome measure. We will also observe the changes in the total scores of the GAD-7 test to provide a direction for further clinical trials. This protocol has been developed according to the SPIRIT 2013 statement and the SPIRIT 2013 explanation and elaboration: guidance for protocols of clinical trials to establish appropriate standards for the scientific, ethical, and safety issues of the study.

However, there are several limitations in this study. One of the major drawbacks is the lack of assessment of the long-term effects of GXJ on primary outcome measures. In our study, the treatment period is only 56 days, which is relatively short. Because of the short length of the follow-up period, the potential roles of GXJ in reducing major vascular events and overall mortality over the long term will remain unknown. Therefore, well-designed randomized controlled trials that compare GXJ with conventional anti-anginal therapies and using longer follow-up periods are required in the future.

\section{Trial status}

Currently, participant recruitment is ongoing.

\section{Additional files}

Additional file 1: GAD-7 (General Anxiety Disorder-7) test. (DOCX $50 \mathrm{~kb}$ )

Additional file 2: SPIRIT checklist. (DOC $121 \mathrm{~kb}$ )

Additional file 3: Research settings. (DOCX $56 \mathrm{~kb}$ )
Additional file 4: CSS Functional Classification of Angina. (DOCX $55 \mathrm{~kb}$ )

Additional file 5: Model consent form [in Chinese]. (DOCX $20 \mathrm{~kb}$ )

\section{Abbreviations}

AE: Adverse event; CAD: Coronary artery disease; CCS: Canadian Cardiovascular Society; CHD: Coronary heart disease; CRF: Case report form; CVD: Cardiovascular disease; ECG: Electrocardiogram; ETT: Exercise tolerance testing; GAD-7: Generalized Anxiety Disorder 7-item (assessment); MACE: Major adverse cardiovascular event; MET: Metabolic equivalent; NCD: Noninfectious chronic disease; PCl: Percutaneous coronary intervention; SF-36: 36-item Short Form Health Survey; TCM: Traditional Chinese medicine

\section{Acknowledgements}

The authors thank those who have participated in the trial. They are also grateful to the staff of the participating centres.

\section{Dissemination policy}

Results will be published in peer-reviewed journals and presented at national and international scientific meetings regardless of the magnitude or direction of effect.

\section{Funding}

This work was supported by the program "New Century Excellent Talents Project of Education Ministry" (NCET-13-0936). The funding parties will play no role in the study design, study conduct, results, or dissemination.

\section{Availability of data and materials}

The datasets generated during the current study are available from the corresponding author upon reasonable request.

\section{Authors' contributions}

WKZ sponsored the study and designed this protocol. JHZ is the primary supervisor and participated in the design of this protocol. YT drafted the manuscript. MYZ and LPZ helped YT draft and revise the manuscript. YQZ and JYM participated in the design of the protocol and are responsible for trial management. RG, XMW, and MJZ are involved in data collection and monitoring for the study. LHM is supervising this study and participated in revising the manuscript. All authors read and approved the final manuscript.

Ethics approval and consent to participate

This trial protocol was approved by the Ethics Committee of the Tianjin University of TCM (ChiCTR1800014258). The study protocol and informed consent are consistent with scientific and ethical requirements. Written informed consent (see Additional file 5) must be obtained from all participants or their legally authorized representatives before enrolment. Study participation is voluntary and can be cancelled at any time without provision of reasons and without negative consequences for their future medical care.

\section{Consent for publication}

We will give informed consent for the publication of the dataset from patients at the point of recruitment to the trial. All the patient details will be fully anonymous.

\section{Competing interests}

The authors declare that they have no competing interests.

\section{Publisher's Note}

Springer Nature remains neutral with regard to jurisdictional claims in published maps and institutional affiliations.

\section{Author details}

${ }^{1}$ Tianjin University of Traditional Chinese Medicine, 312 Anshanxi Road, Nankai District, Tianjin 300193, China. ${ }^{2}$ The Second Hospital Affiliated to Tianjin University of Traditional Chinese Medicine, 69 Zengchan Road, Hebei District, Tianjin 300010, China. ${ }^{3}$ The First Hospital Affiliated to Tianjin University of Traditional Chinese Medicine, 314 Anshanxi Road, Nankai District, Tianjin 300193, China. ${ }^{4}$ Xiyuan Hospital of Chinese Academy of Traditional Chinese Medicine, Haidian District, Beijing 100091, China. ${ }^{5}$ Peking University First Hospital, 8 Xishiku Road, Xicheng District, Beijing 1000343, 
China. ${ }^{6}$ The First Hospital Affiliated to Henan University of Traditional Chinese Medicine, 19 Renmin Road, Zhengzhou City 450000, Henan, China. ${ }^{7}$ Fuwai Hospital, National Center for Cardiovascular Diseases, 167 Beilishi Road, Xicheng District, Beijing 100037, China.

\section{Received: 5 February 2018 Accepted: 28 September 2018}

Published online: 20 October 2018

\section{References}

1. kalidis El, Vardas PE. Guidelines on the management of stable angina pectoris. Eur Heart J. 2006;27(21):2606-7.

2. Ma D, Sakai H, Wakabayashi $\mathrm{C}$, et al. The prevalence and risk factor control associated with noncommunicable diseases in China, Japan, and Korea. J Epidemiol. 2017;27(12):568-73.

3. Mozaffarian D, Benjamin EJ, Go AS, Arnett DK, Blaha MJ, Cushman M, et al. Heart disease and stroke statistics-2016 update: a report from the American Heart Association. Circulation. 2016;133(4):e38-360. https://doi. org/10.1161/CIR.

4. Genders TSS, Hunink MGM. Epidemiology of coronary artery disease. In: Cademartiri F, Casolo G, Midiri M, editors. Clinical applications of cardiac CT. Milan: Springer; 2012. p. 23-7.

5. National Institutes of Health National Heart, Lung, and Blood Institute. Morbidity \& Mortality: 2012 chart book on cardiovascular, lung, and blood diseases. Bethesda: National Heart, Lung, and Blood Institute; 2012.

6. Gao ZY, Xu H, Shi DZ, et al. Analysis on outcome of 5284 patients with coronary artery disease: the role of integrative medicine. J Ethnopharmacol. 2012;141 (2):578-83.

7. Chen KJ, Shi DZ, Xu H, Lv SZ, Li TC, Ke YN, et al. XS0601 reduces the incidence of restenosis: a prospective study of 335 patients undergoing percutaneous coronary intervention in China. Chin Med J. 2006;119:6-13.

8. Ge YB, Mao JY. Analysis of distribution of TCM syndromes in 7512 patients with coronary heart diseases. Shandong J Tradit Chin Med. 2011:30(04):227-9.

9. Zhag $S, L I Z, C a o L$, et al. Meta-analysis of reinforcing Qi and activating blood recipe for coronary eart disease. J Cardiac. 2017;29(03):307-12.

10. Xiong XJ, Wang Z, Wang J. Innovative strategy in treating angina pectoris with Chinese patent medicines by promoting blood circulation and removing blood stasis: experience from combination therapy in Chinese medicine. Curr Vasc Pharmacol. 2014;10:15.

11. Zhang GQ, Wang YY, Yan GZ. Research on the pharmacodynamics of Guanxinjing oral liquid. J Hebei Med Coll. 1995;16(02):73-4.

12. Song SJ, Wei FJ, Wei TY, et al. The effect of Guanxinjing capsule on the treatment of qi deficiency and blood stasis type coronary heart disease. China Pharm Econ. 2014;S1:133134.

13. de Jonge P, Spijkerman TA, van den Brink RH, Ormel J. Depression after myocardial infarction is a risk factor for declining health related quality of life and increased disability and cardiac complaints at 12 months. Heart. 2006;92(1):32-9.

14. Meijer A, Conradi HJ, Bos EH, et al. Prognostic association of depression following myocardial infarction with mortality and cardiovascular events: a meta-analysis of 25 years of research. Gen Hosp Psychiatry. 2011;33(3):203.

15. Barth J, Schumacher M, Herrmann-Lingen C. Depression as a risk factor for mortality in patients with coronary heart disease: a meta-analysis. Psychosom Med. 2004;66(6):802-13.

16. Huffman JC, Celano CM, Januzzi JL. The relationship between depression, anxiety, and cardiovascular outcomes in patients with acute coronary syndromes. Neuropsychiatr Dis Treat. 2010;6(6):123-36.

17. Celano CM, Suarez L, Mastromauro C, Januzzi JL, Huffman JC. Feasibility and utility of screening for depression and anxiety disorders in patients with cardiovascular disease. Circ Cardiovasc Qual Outcomes. 2013;6(4):498-504.

18. Smith DF, Sterndorff B, Røpcke G, et al. Prevalence and severity of anxiety, depression and Type A behaviors in angina pectoris. Scand J Psychol. 2010; 37(3):249-58.

19. Trivedi R, Gerrity M, Rumsfeld JS, et al. Angina symptom burden associated with depression status among veterans with ischemic heart disease. Ann Behav Med. 2015;49(1):58-65.

20. Jespersen L, Abildstrøm SZ, Hvelplund A, et al. Persistent angina: highly prevalent and associated with long-term anxiety, depression, low physical functioning, and quality of life in stable angina pectoris. Clin Res Cardiol. 2013;102(8):571-81.
21. Carney RM, Blumenthal JA, Freedland KE, Youngblood M, et al. Depression and late mortality after myocardial infarction in the Enhancing Recovery in Coronary Heart Disease (ENRICHD) study. Psychosom Med. 2004;66(4):466.

22. Lesperance F, Frasure-Smith N, Koszycki D, et al. Effects of citalopram and interpersonal psychotherapy on depression in patients with coronary artery disease: The Canadian Cardiac Randomized Evaluation of Antidepressant and Psychotherapy Efficacy (CREATE) Trial. JAMA. 2007;297:367-79.

23. Honig A, Kuyper AMG, Schene AH, et al. Treatment of post-myocardial infarction depressive disorder: a randomized, placebo-controlled trial with mirtazapine. Psychosom Med. 2007:69:606-13.

24. Liu HX. Report of the research on the treatment mechanism of Guanxinjing capsule in myocardial infarction with depression disorder. Chin J Evid Based Cardiovasc Med. 2013;5(04):434.

25. Wang X, Qin Z, Zhao ZF. A consensus on the diagnosis and treatment of depression before and after percutaneous coronary intervention (PCI). J Tradit Chin Med. 2015;56(04):357-60.

26. Wang L, Lu K, Wang CY, et al. Analysis of reliability and validity of GAD-2 and GAD-7 in cardiovascular anxiety screening. Sichuan Mental Health. 2014;27(03):198-201.

27. Spitzer RL, Kroenke K, Williams JB, et al. A brief measure for assessing generalized anxiety disorder: the GAD-7. Arch Intern Med. 2006;166(10): 1092-7.

28. Cheng C-W, Tai-xiang W, Shang H-c, et al. CONSORT extension for Chinese herbal medicine formulas 2017: recommendations, explanation, and elaboration. Ann Intern Med. 2017;167(2):112-21.

29. Cardiology Branch of Chinese Medical Association. The guideline on diagnosis and treatment of chronic stability angina. Chin I Cardiovasc Dis. 2007;35;(03):195-206.

30. Task Force Members, Montalescot G, Sechtem U, et al. 2013 ESC guidelines on the management of stable coronary artery disease: the Task Force on the management of stable coronary artery disease of the European Society of Cardiology. Eur Heart J. 2013;34(38):2949-3003.

31. Healthcare Improvement Scotland. Management of stable angina. Coronary revascularisation in the management of stable angina pectoris (SIGN Guideline). Edinburgh: Healthcare Improvement Scotland; 2018-04/2018-0604. http://www.sign.ac.uk.

32. Campeau L. Letter: grading of angina pectoris. Circulation. 1976;54:522-3.

33. China Food and Drug Administration. Technical guidelines for clinical research on the treatment of angina pectoris by traditional Chinese medicine and natural medicine. http://www.sda.gov.cn/WS01/CL1616/ 90942.html.2011.07.08.

34. Fletcher GF, Ades PA, Kligfield P, et al. Exercise standards for testing and training: a scientific statement from the American Heart Association. Circulation. 2013;128(8):873-934.

35. World Health Organization. Global status report on noncommunicable diseases 2014. Women. 2014;47(26):2562-3.

36. Wang XL, Mao JY, Hou YZ. Preliminary study of establishing clinical effect evaluation methods of Chinese medicine based on combination of disease and syndrome, systematic staging, and multi-dimension index. Chin J Integr Tradit West Med. 2013;33(2):270.

Ready to submit your research? Choose BMC and benefit from:

- fast, convenient online submission

- thorough peer review by experienced researchers in your field

- rapid publication on acceptance

- support for research data, including large and complex data types

- gold Open Access which fosters wider collaboration and increased citations

- maximum visibility for your research: over $100 \mathrm{M}$ website views per year

At BMC, research is always in progress.

Learn more biomedcentral.com/submissions 